\title{
Differential reinstatement predicted by preextinction response rate
}

\author{
ADAM H. DOUGHTY \\ West Virginia University, Morgantown, West Virginia \\ PHIL REED \\ University of Wales, Swansea, Wales \\ and \\ KENNON A. LATTAL \\ West Virginia University, Morgantown, West Virginia
}

\begin{abstract}
Reinstatement refers to the recovery of previously extinguished responding by the responseindependent delivery of a stimulus that was a reinforcer in training. Two experiments were conducted to examine relative reinstatement following the training of differential preextinction response rates, either with equal (Experiment 1) or unequal (Experiment 2) preextinction reinforcement rates. In Experiment 1, each of 3 pigeons first pecked at relatively high rates in the tandem variable-time 117-sec fixed-interval 3-sec component of a multiple schedule and at lower rates in a separate tandem variableinterval 117-sec fixed-time 3-sec component. Reinforcement rates were equal between components. Pecking then was extinguished in each component, before being reinstated under a multiple variabletime 120-sec variable-time 120-sec schedule. Greater reinstatement occurred in the component previously correlated with higher rates of pecking. In Experiment 2, in an initial condition, the mean rate of lever pressing for one group of 8 rats was significantly higher under a fixed-ratio 3 schedule than for another group of 8 rats under a fixed-ratio 1 schedule. Mean reinforcement rate was significantly higher for the group exposed to the fixed-ratio 1 schedule. For each group, lever pressing then was extinguished, before being reinstated under a variable-time 30-sec schedule. Significantly greater mean reinstatement occurred for the group previously exposed to the fixed-ratio 3 schedule. These results suggest that differential reinstatement may be predicted by preextinction response rate, perhaps independently of preextinction reinforcement rate.
\end{abstract}

Reinstatement is studied in several areas of psychology. Despite procedural variations across these different areas, reinstatement can be studied using an experimental procedure involving three phases (cf. Reid, 1957). In the first, responding is acquired and maintained by reinforcement. In the second, reinforcer delivery is eliminated, and responding subsequently ceases. In the third, responding recovers when the reinforcer from training is delivered response independently. Below we discuss how the present research involving reinstatement with pigeons and rats responding under operant procedures might be of interest to researchers studying reinstatement in other areas of psychology.

Reinstatement has been studied often in behavioral pharmacology (e.g., de Wit \& Stewart, 1981), infant learning and cognition (e.g., Rovee-Collier, Sullivan, Enright,

The first author received support, while writing portions of this manuscript, from Institutional National Research Service Award 18852 awarded to the University of Kansas. Address correspondence and reprint requests to A. H. Doughty, University of Kansas, Parsons Research Center, P.O. Box 738, 2601 Gabriel, Parsons, KS 67357 (e-mail: adoughty@ku.edu).
Lucas, \& Fagen, 1980), and respondent conditioning (e.g., Bouton \& Bolles, 1979). In behavioral pharmacology, much of the research involving reinstatement has focused on its role as a model of relapse (see Shaham, Shalev, Lu, de Wit, \& Stewart, 2003, for a review). For example, de Wit and Stewart initially reinforced (with cocaine) the responding of rats. Responding decreased when cocaine delivery was discontinued (i.e., extinction). Responding then was reinstated by the response-independent delivery of cocaine and other drugs from the same pharmacological class (e.g., amphetamine), whereas this response recurrence did not occur with drugs outside that class (e.g., ethanol). Rovee-Collier et al. first reinforced (with mobile activation) the foot kicking of infants. Seven days after this training, the infants were separated into two groups. Each infant in one group received response-independent exposure to mobile activation, whereas infants in the other group were not exposed to the mobile. The next day, each infant was exposed to the mobile (without reinforcement), and significantly more responding occurred for the group that previously received the response-independent mobile activation. Finally, Bouton and Bolles first conditioned fear to a neutral stimulus (correlated with shock) in a 
conditioned-suppression paradigm. After extinguishing this fear, it reappeared later when shocks were delivered response independently. Thus, the study of reinstatement has generated an interest among a variety of researchers because of its relevance to a number of important phenomena (i.e., drug seeking and treatment, memory mechanisms involved in the retention of learning, or methods for improving behavior therapy techniques).

Reinstatement also has been examined with animals in the operant laboratory (e.g., Franks \& Lattal, 1976). Such examination is noteworthy because of the rising interest in the effects of remote reinforcement contingencies (i.e., behavioral history) on current responding (cf. Lieving \& Lattal, 2003). For example, Franks and Lattal studied the effects on reinstatement of preceding extinction with two different reinforcement schedules. Prior to extinction, in some conditions rats responded at higher rates (with a higher reinforcement rate) under a variable-ratio (VR) schedule, whereas in other conditions, the same rats responded at lower rates (with a lower reinforcement rate) under a differential-reinforcementof-low-rate (DRL) schedule. When food was delivered response independently after extinction, differential reinstatement occurred in that there were higher response rates after VR-schedule training than after DRL-schedule training. Thus, because responding always was at or near zero prior to being reinstated, the differential reinstatement must be attributed to the remote reinforcement contingencies that were in effect prior to extinction.

The purpose of the present two experiments was to explore further the conditions that generate differential reinstatement and to suggest how such exploration might be relevant to other researchers investigating reinstatement. In Franks and Lattal (1976), the greater reinstatement that followed the VR schedule relative to the DRL schedule could have resulted from the higher response rate generated by the VR schedule, the higher reinforcement rate under that schedule, or both. Thus, the present experiments sought to determine whether differential reinstatement would occur following the training of unequal preextinction response rates in which the higher response rate was correlated with the same reinforcement rate as was the lower one (Experiment 1) or in which the higher response rate was correlated with a lower reinforcement rate (Experiment 2).

\section{EXPERIMENT 1}

The differential reinstatement reported by Franks and Lattal (1976) could have resulted from differences in preextinction response rate, preextinction reinforcement rate, or both. In addition, Franks and Lattal observed their effects across, rather than within, conditions. Experiment 1 had two aims: to investigate the utility of a multiple schedule for demonstrating differential reinstatement with individual organisms in the same session, and to examine the role of preextinction response and reinforce- ment rates in generating differential reinstatement. With reinforcement rates equated, differential response rates first were established in separate multiple-schedule components, before extinction and response-independent food delivery. The issue, then, was whether differential reinstatement would follow the training of differential preextinction response rates when reinforcement rates were equated.

\section{Method}

Subjects. Three White Carneau pigeons with experimental histories of food-maintained key pecking were at approximately $80 \%$ of their free-feeding body weights. Each was housed individually, with water and health grit continuously available in the home cage. Each was given supplemental feeding, if necessary, to maintain its target weight. Lights were on in the home colony from 7 am to $7 \mathrm{pm}$.

Apparatus. A standard operant conditioning chamber, with a work area $35 \mathrm{~cm}$ high $\times 30 \mathrm{~cm}$ wide $\times 30 \mathrm{~cm}$ long, was enclosed in a sound- and light-attenuating box. Two response keys $(2-\mathrm{cm}$ diameter) were located on the front wall $25 \mathrm{~cm}$ above the floor and $10 \mathrm{~cm}$ from either side wall and could be transilluminated green or red. General illumination was provided throughout the session, except during 15-sec blackouts (see below) and reinforcer delivery, by white light from the lower right corner of the front wall. The keylights also were darkened during reinforcer delivery, which consisted of 3-sec access to mixed grain provided in a food hopper located behind a $5-\mathrm{cm}^{2}$ aperture centered on the front wall with its lower edge $5 \mathrm{~cm}$ above the floor. The aperture was illuminated only during food delivery. Programming and data recording were controlled by a computer in an adjacent room using MED-PC software (MED Associates, Inc., Georgia, VT).

Procedure. Due to the history of each pigeon, the experiment commenced without pretraining. A two-component multiple schedule operated in which each component was $3 \mathrm{~min}$ in duration and was preceded by a 15 -sec period with the lights turned off. In one component, the left key was green and the right key was dark. In the other component, the right key was red and the left key was dark. The first component of each session was chosen randomly, and the remainder of the components strictly alternated thereafter until each occurred 10 times. Table 1 shows the number of sessions per condition for each pigeon. Sessions occurred 7 days per week.

In the first condition, a tandem variable-time (VT) 117-sec fixedinterval (FI) 3-sec schedule was correlated with the green key. That is, after a variable period of time averaging $117 \mathrm{sec}$, the FI schedule began (in the absence of a stimulus change) such that after a 3sec interval, the first keypeck produced food. Thus, pecking the green key was maintained by immediate reinforcement. Also in the first condition, a tandem variable-interval (VI) 117-sec fixed-time (FT) 3-sec schedule was correlated with the red key. That is, the first keypeck after a variable period of time averaging $117 \mathrm{sec}$ initiated a 3-sec period (in the absence of a stimulus change) after which food was delivered response independently. Thus, pecking the red

Table 1

Number of Sessions for Each Pigeon During Each Condition of Experiment 1

\begin{tabular}{cccc}
\hline Condition & Pigeon 1496 & Pigeon 7126 & Pigeon 9091 \\
\hline 1 & 45 & 25 & 44 \\
2 & 27 & 46 & 45 \\
3 & 8 & 8 & 8 \\
\hline
\end{tabular}

Note-Condition 1 refers to the multiple tandem VT FI tandem VI FT schedule. Conditions 2 and 3 refer, respectively, to the multiple extinction extinction schedule and the multiple VT VT schedule. 
key was maintained by unsignaled delayed reinforcement. The VI and VT schedules comprised the same 20 intervals derived from the distribution described by Fleshler and Hoffman (1962). This condition lasted a minimum of 25 sessions and until there was no systematically increasing or decreasing trend in response rate in either component for at least 6 consecutive sessions, as assessed visually.

In the second condition, extinction replaced the reinforcement schedules just described, and all other details were identical to the first condition. For each pigeon, the second condition continued until 10 consecutive sessions occurred with fewer than 10 pecks in each component. The final condition was a multiple VT 120 -sec VT 120-sec schedule, such that food was delivered response independently in each component on an average of once every $120 \mathrm{sec}$. All other details were as in the first two conditions, and this final condition continued for 8 sessions.

\section{Results}

Figure 1 shows the number of responses per session in each component in the final six sessions of the first condition and the total number of responses in each session of the second and third conditions. The results of each condition are separated by solid vertical lines. The range of values on the $y$-axis is different for each pigeon, but the results for each were similar. In the first condition, response rate was higher with immediate reinforcement than with unsignaled delayed reinforcement. In the second condition, responding progressively declined to near zero in each component. In the third condition, responding recurred in each component, with more responding in the component previously correlated with immediate reinforcement.

Obtained reinforcement rate (i.e., reinforcers per minute) was calculated for the final six sessions of the first condition. For Pigeons 1496, 7126, and 9091, respectively, these mean rates were $0.45,0.47$, and 0.45 with immediate reinforcement and $0.46,0.45$, and 0.46 with unsignaled delayed reinforcement. Reinforcement rate also was similar between components in the other
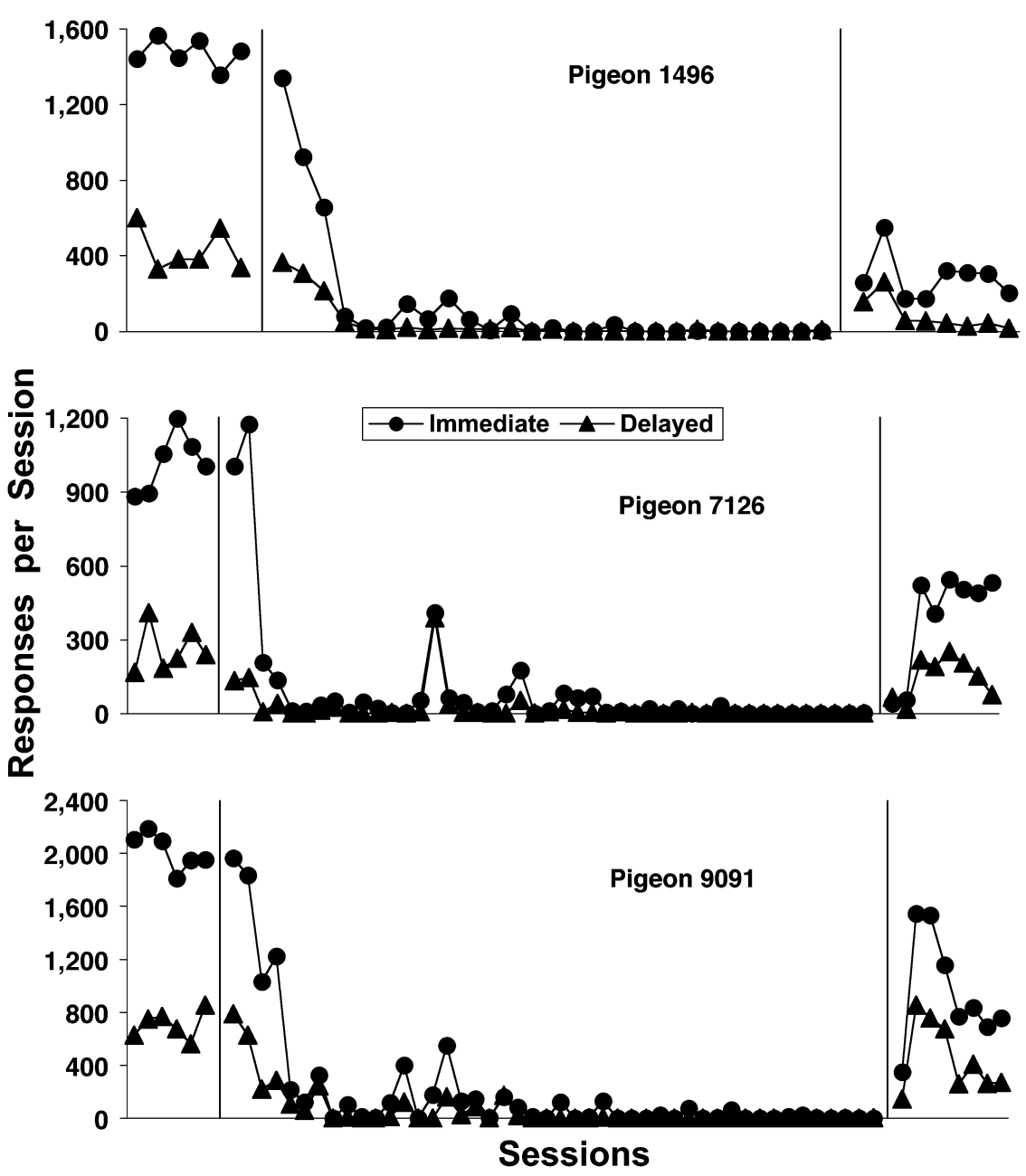

Figure 1. Number of responses per session in the final six sessions of the first condition and the total number of responses in each session of the second and third conditions for each component and each pigeon in Experiment 1. 
conditions because of the operation of the identical extinction and VT schedules.

\section{Discussion}

For each pigeon, responding maintained under different schedules and extinguished to equivalent low levels was reinstated by the response-independent delivery of food, replicating several earlier findings (e.g., Reid, 1957). Also demonstrated was the utility of a multiple schedule to study differential reinstatement with individual organisms in the same session. With reinforcement rate equal, the establishment of different preextinction response rates was sufficient to generate differential reinstatement, with the higher preextinction response rate reinstated to a higher level. Thus, this result suggests that the differential reinstatement reported by Franks and Lattal (1976) probably was a function of the differential response rates arranged by the VR and DRL schedules, as opposed to the discrepant reinforcement rates arranged by those schedules.

\section{EXPERIMENT 2}

The second experiment further assessed the findings of Experiment 1. In both Franks and Lattal (1976) and Experiment 1, greater reinstatement occurred following the training of higher response rates, whether reinforcement rate was greater also (as in Franks \& Lattal's study) or equal (as in Experiment 1). In Experiment 2, therefore, reinstatement was compared under conditions in which a higher response rate was correlated with a lower reinforcement rate. The generality of the results of Experiment 1 was assessed in Experiment 2 in two additional ways. First, rats were used as subjects instead of pigeons, and second, a between-subjects rather than a within-subjects design was used.

\section{Method}

Subjects. Sixteen male, Lister hooded rats with histories of foodmaintained lever pressing were at $85 \%$ of their free-feeding body weights. The rats were housed in groups of 4 and were provided constant access to water in the home cage.

Apparatus. Four identical operant conditioning chambers were used. Each chamber was located in a sound- and light-attenuating box and was equipped with a ventilation fan providing a background masking noise of $65 \mathrm{~dB}$ (A). Each chamber contained two identical response levers located on each side of a centrally located food tray. The food tray was covered by a hinged, clear Perspex flap, behind which 45-mg Noyes food pellets were delivered. A jeweled light was positioned $3 \mathrm{~cm}$ above each lever.

Procedure. Due to the history of each rat, the experiment commenced without pretraining. Each session began with the illumination of the jeweled light above the left lever, and the light was turned off at the end of each session. The rats were divided randomly into two groups of 8 . In the first condition, for each rat in one group, pressing the left lever was reinforced according to a fixed-ratio (FR) 1 schedule. For each rat in the other group, an FR 3 schedule was correlated with the left lever. Throughout each condition, responses to the right lever were recorded but had no programmed consequences. Each session continued until 30 food deliveries occurred. Sessions were conducted 7 days per week, and this initial condition lasted 10 sessions.

In the next condition, extinction was in effect for each rat in each group, such that food never was delivered. Each session lasted $10 \mathrm{~min}$, and all other details were the same as in the first condition. This second condition lasted nine sessions. The final condition consisted of one 30-min session for each rat. In this session, a VT 30-sec schedule was in effect, such that food was delivered on the average of once every $30 \mathrm{sec}$ independently of responding.

\section{Results}

Figure 2 shows mean response rate for each group in each session. For statistical analyses, in which a rejection criterion of $p<.05$ was adopted, the final session of each of the first two conditions and the one session of the third condition were examined. In the last session of the first condition, mean response rate was significantly

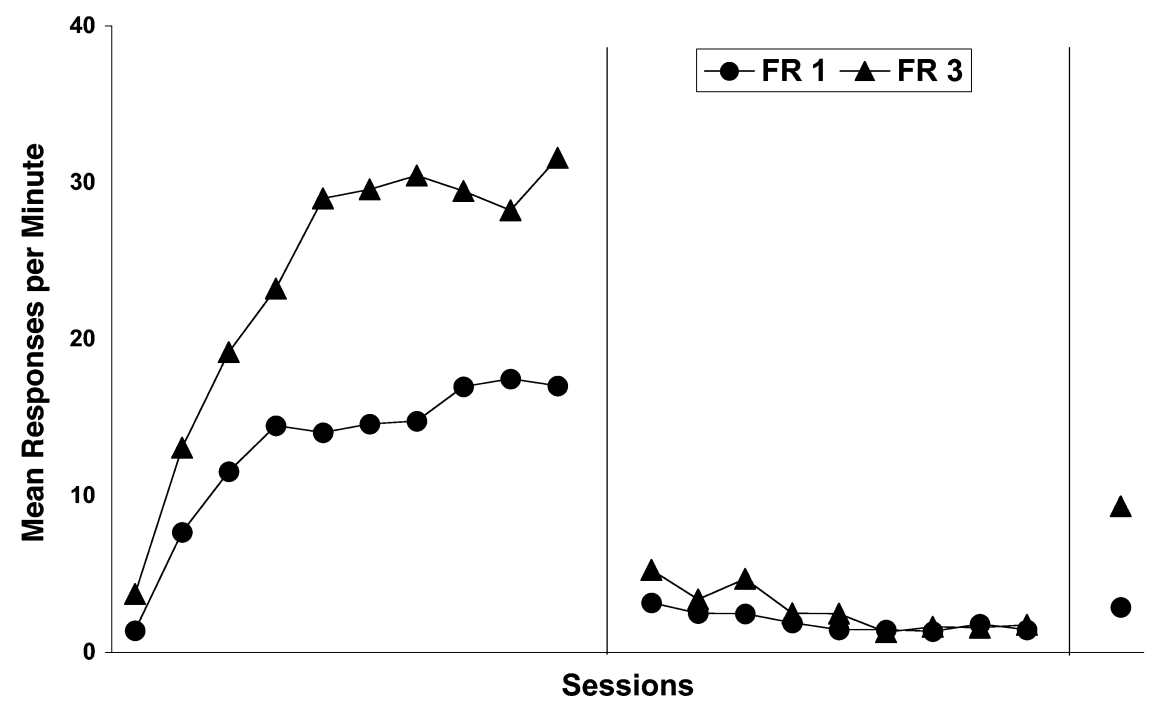

Figure 2. Mean responses per minute for each group of rats in each session across the three conditions of Experiment 2. 
higher for the group exposed to the FR 3 schedule than for the group exposed to the FR 1 schedule $[t(7)=5.34]$. There was no significant difference in mean response rates between the groups in the last session of the second condition $[t(7)=0.57]$. In the third condition, mean response rate again was significantly higher for the group exposed to the FR 3 schedule than for the group exposed to the FR 1 schedule $[t(7)=6.48]$.

Obtained mean reinforcement rate (i.e., reinforcers per minute) also was calculated for the two groups during the sessions shown in Figure 2. In the last session of the first condition, mean reinforcement rate was significantly higher for the group exposed to the FR 1 schedule $(16.98 / \mathrm{min})$ than for the group exposed to the FR 3 schedule $(10.52 / \mathrm{min})[t(7)=5.77]$. Because both groups underwent extinction and identical VT 30-sec schedules in the second and third conditions, respectively, there were no differences in mean reinforcement rate between the groups in either of the final two conditions.

As noted above, the number of responses to the right lever was also recorded in each session. In the last session of the first condition, there were no such responses for either group. In the last session of the second condition, there were a total of 12 and 18 such responses for the groups exposed to the FR 1 and FR 3 schedules, respectively. In the one session of the third condition, there were a total of 10 and 19 such responses for the groups exposed to the FR 1 and FR 3 schedules, respectively.

\section{Discussion}

Previously extinguished food-maintained responding was reinstated by the response-independent delivery of food, replicating several previous findings (e.g., Experiment 1). The results also demonstrate the generality of the findings of Experiment 1 by the use of a different species (i.e., rats) and experimental design (i.e., betweensubjects design). Most significantly, the results further confirm the importance of differences in preextinction response rates in the production of differential reinstatement, since the previously higher response rate was reinstated more than the previously lower response rate, despite the latter being correlated with a higher reinforcement rate.

\section{GENERAL DISCUSSION}

There were several procedural differences between the present two experiments. The first consisted of pigeons pecking keys in different multiple-schedule components, whereas in the second, rats pressed levers in a betweensubjects design. In addition, the differential preextinction response rates in the first were correlated with the same reinforcement rate, whereas the higher preextinction response rate in the second experiment was correlated with a lower reinforcement rate. Nonetheless, a similar result occurred in each-greater reinstatement of a formerly higher response rate.

That differential reinstatement occurred in each experiment illustrates that the study of reinstatement can aid the understanding of behavioral history. That is, despite responding being at equivalent low rates across multiple-schedule components (Experiment 1) or groups (Experiment 2) at the onset of response-independent food delivery, that delivery produced different effects on responding. Those effects only could be predicted by considering the (remote) reinforcement schedule training each pigeon or rat had received earlier. More specifically, to predict the relative reinstatement observed one only had to consider preextinction response rates. Thus, the present results are noteworthy in that they help to isolate further the conditions controlling differential reinstatement.

Differential reinstatement, here, simply describes the result that responding occurred at different rates across components or groups during the condition involving response-independent food delivery. To say, therefore, that there was greater reinstatement following immediate reinforcement in Experiment 1 and after the FR 3 schedule in Experiment 2 does not involve comparing response rates relative to subjects' training level. One might, however, argue that such a comparison would be more appropriate than the analysis we have chosen in the present study. Consider the results for Pigeon 7126, for example: Because during the final condition, response rates in the component with unsignaled delayed reinforcement were closer to their training level than were response rates in the other component, one might argue that this result should be labeled greater reinstatement. There are two reasons, however, why it seems more meaningful to describe relative reinstatement in terms of its change from the preceding extinction condition (i.e., from zero or near-zero responses) as opposed to its change from the original training level. First, from a practical standpoint, it seems more meaningful to consider the current rate of response recovery, as opposed to its change from pretreatment level, when the recovering response is "drug seeking" (e.g., Shaham et al., 2003) or some other clinical relapse (e.g., Bouton, 2002). Second, there are several reasons why relatively high response rates would not be reinstated to their training level, making a comparison to that level, in some sense, meaningless. For example, with all other things being equal, response rates almost always are higher under response-dependent food delivery than under response-independent food delivery (e.g., Sizemore \& Lattal, 1977). In addition, whereas the relatively high response rates occurring at the end of training followed several sessions of response-dependent food delivery, the condition of response-independent food delivery followed several sessions of extinction (little to no responding and no reinforcement).

It should not be concluded from the present discussion that preextinction reinforcement rate does not exert any effect on subsequent, differential reinstatement. A noteworthy test of the role, if any, of preextinction reinforcement rate would arrange for the reinstatement of formerly equivalent response rates that were supported by different reinforcement rates.

That the differential reinstatement observed in the present experiments could be predicted solely by con- 
sidering preextinction response rates may have implications for researchers in other areas of psychology who are interested in reinstatement. Recently, it was noted that "an important question ... is whether the rate of responding during training leads to qualitative differences ... during testing" and that "despite the fact that only several studies have addressed this issue ... it is not likely . . . a major methodological concern" (Shalev, Grimm, \& Shaham, 2002, p. 31). On the one hand, the present results suggest that it is imperative for researchers to consider training response rate if the research goal is to describe the relative degree of reinstatement across manipulations (e.g., drug type). On the other hand, if the goal is only to address the question of whether some drug (or drug-correlated stimulus), delivered response independently, will reinstate responding, then training response rate probably is not as important to consider. Nonetheless, it still may prove beneficial in such studies to generate a relatively high training response rate to detect better any potential reinstatement.

The present results also may bear on the study of reinstatement with infants ${ }^{1}$ (e.g., Rovee-Collier et al., 1980). The reinforcement schedule most commonly used to establish responding with infants operates such that a mobile is activated whenever the infant is kicking. Thus, in such studies, the effects of preextinction response and reinforcement rates to our knowledge have not been disentangled. A noteworthy extension of the present report would be to mimic the conditions of Experiment 2 with infants. For one group, a higher response rate could be correlated with a lower reinforcement rate, whereas for a different group a lower response rate could be correlated with a higher reinforcement rate (i.e., intermittent reinforcement could be arranged for one or both groups). Following such training, and after all the infants received an equivalent response-independent exposure to the training reinforcer, the degree of response recovery could be compared across groups in a final condition. Isolating response and reinforcement rates in such a paradigm might aid in understanding the mechanism(s) by which reinstatement occurs.

Regarding such mechanism(s), the present results are described well by an interpretation of reinstatement in terms of the discriminative function of food delivery established during training (e.g., Franks \& Lattal, 1976). That is, during initial training, food delivery not only selected, or strengthened, the responding that preceded it, but it also was discriminative for subsequent responding of a similar form (i.e., rate). In Experiment 1, food delivery was discriminative for different response rates according to which antecedent stimulus (i.e., keylight color) was present (i.e., which multiple-schedule component was in effect). This discriminative stimulus function persisted across extinction and exerted control of responding after food again was delivered (response independently) in the final condition. The interpretation just described is not radically different from the associationist interpretation that often is used to describe the stimulus control of response-recovery phenomena (e.g., Bouton, 2002). In such associationist terms, reinstatement occurs because the response-independent outcome (food) reinstates the originally established stimulus-response association (e.g., in Experiment 1, the relationship between keylight color and pecking at a particular rate). In other words, extinction did not destroy the stimulus-outcome associations established during training (see also Rescorla, 2003). Given the resemblance between the two aforementioned interpretations, another step in describing reinstatement from an interdisciplinary viewpoint would be to consider the relation between these interpretations and research involving the neurobiological underpinnings of reinstatement (e.g., Shalev et al., 2002).

\section{REFERENCES}

Bouton, M. E. (2002). Context, ambiguity, and unlearning: Sources of relapse after behavioral extinction. Biological Psychiatry, 51, 976-986.

Bouton, M. E., \& Bolles, R. C. (1979). Role of conditioned contextual stimuli in reinstatement of extinguished fear. Journal of Experimental Psychology: Animal Behavior Processes, 5, 368-378.

DE WIT, H., \& STEWART, J. (1981). Reinstatement of cocaine-reinforced responding in the rat. Psychopharmacology, 75, 134-143.

Fleshler, M., \& HoffmAN, H. S. (1962). A progression for generating variable-interval schedules. Journal of the Experimental Analysis of Behavior, 5, 529-530.

FrANKS, G. J., \& LATTAL, K. A. (1976). Antecedent reinforcement schedule training and operant response reinstatement in rats. Animal Learning \& Behavior, 4, 374-378.

Hildreth, K., Sweeney, B., \& Rovee-Collier, C. (2003). Differential memory-preserving effects of reminders at 6 months. Journal of Experimental Child Psychology, 84, 41-62.

LiEVING, G. A., \& LATTAL, K. A. (2003). Recency, repeatability, and reinforcer retrenchment: An experimental analysis of resurgence. Journal of the Experimental Analysis of Behavior, 80, 217-233.

REID, R. L. (1957). The role of the reinforcer as a stimulus. British Journal of Psychology, 49, 292-309.

Rescorla, R. A. (2003). Protection from extinction. Learning \& Behavior, 31, 124-132.

Rovee-Collier, C. K., Sullivan, M. W., Enright, M., Lucas, D., \& Fagen, J. W. (1980). Reactivation of infant memory. Science, 208, 1159-1161.

Shaham, Y., Shalev, U., Lu, L., De Wit, H., \& Stewart, J. (2003). The reinstatement model of drug relapse: History, methodology and major findings. Psychopharmacology, 168, 3-20.

Shalev, U., Grimm, J. W., \& Shaham, Y. (2002). Neurobiology of relapse to heroin and cocaine seeking: A review. Pharmacological Reviews, 54, 1-42.

Sizemore, O. J., \& LatTal, K. A. (1977). Dependency, temporal contiguity, and response-independent reinforcement. Journal of the Experimental Analysis of Behavior, 27, 119-125.

\section{NOTE}

1. The term reactivation, as used in the infant learning and cognition literature, more closely resembles the procedures discussed herein than does the term reinstatement. In that literature, reinstatement resembles what typically is called reconditioning in the animal learning literature (e.g., Hildreth, Sweeney, \& Rovee-Collier, 2003).

(Manuscript received August 13, 2003; revision accepted for publication January 6, 2004.) 